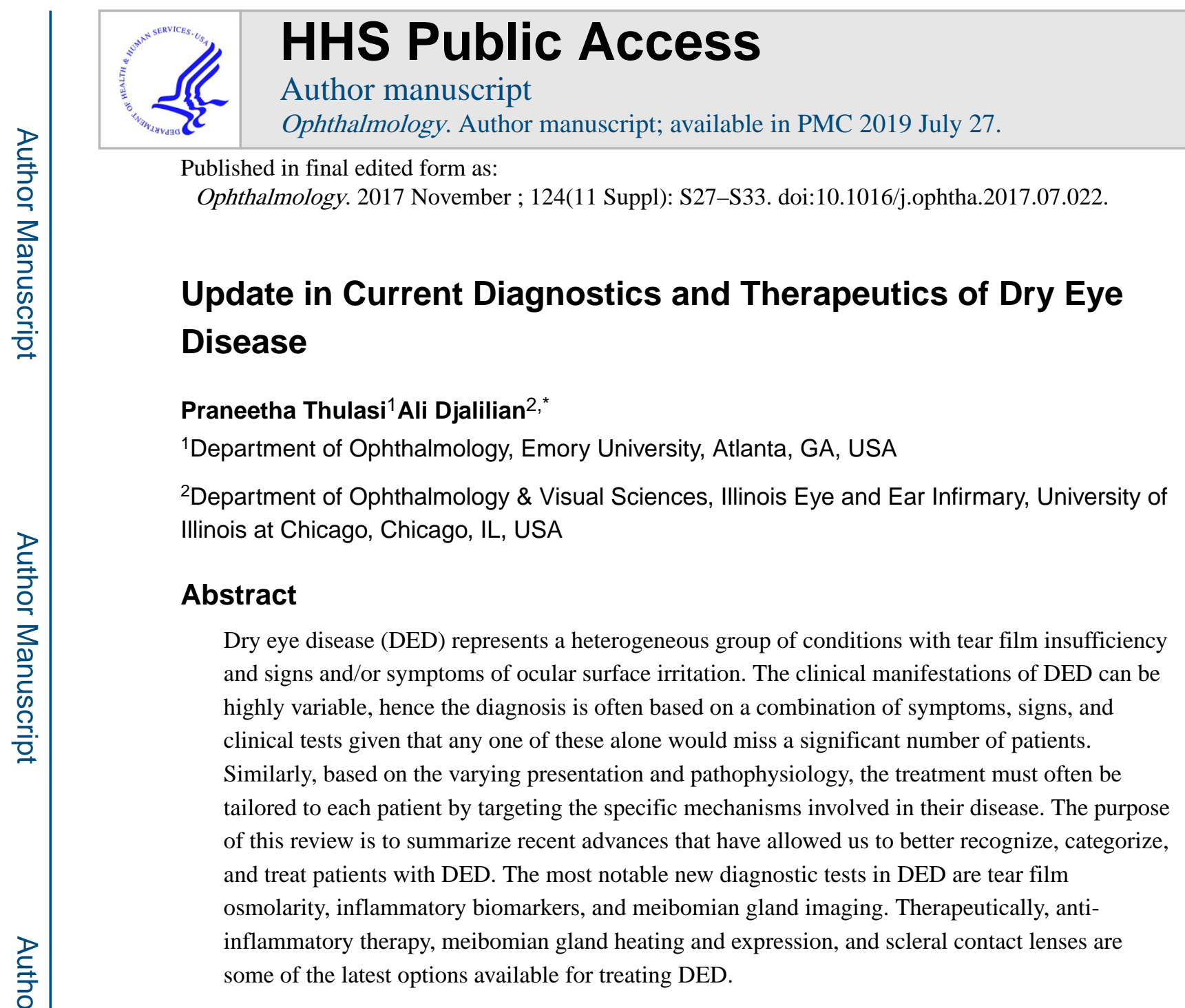

\title{
Introduction
}

Dry eye disease (DED) has multifactorial etiologies and pathophysiologies that ultimately lead to tear film insufficiency and signs and/or symptoms of ocular surface disease. The clinical manifestations of DED often have poor correlation between signs and symptoms. Likewise, diagnostic tests of the ocular surface often have significant variability. Thus, the diagnosis of DED is typically based on a combination of symptoms, signs, and clinical tests since any one of these alone would miss a number of patients. Similarly, there is no single therapeutic strategy that fits all patients and instead, treatment is best individualized by targeting the specific mechanisms that are driving the disease process in each patient. The purpose of this review is to summarize recent advances that have allowed us to better recognize, categorize, and manage patients with DED. In particular, the emphasis is placed on the technology without specifically endorsing or recommending any particular product.

*Corresponding author thulasip@gmail.com, adjalili@uic.edu, 312-996-8936.

Publisher's Disclaimer: This is a PDF file of an unedited manuscript that has been accepted for publication. As a service to our customers we are providing this early version of the manuscript. The manuscript will undergo copyediting, typesetting, and review of the resulting proof before it is published in its final citable form. Please note that during the production process errors may be discovered which could affect the content, and all legal disclaimers that apply to the journal pertain.

Conflict of Interest

None 


\section{Diagnostic Testing}

While clinical history and examination remain the mainstay of DED diagnostics, ancillary testing with newer imaging technology has added much to our armamentarium. Many of these are available as point-of-care tests, making them widely available to clinicians. An important point to reiterate is that since DED is a heterogeneous disease, the tests described below may be useful for some subtypes of DED, but not all. Therefore, the results of each test should be interpreted in the context of each patient and not as an absolute measure of whether a patient has DED.

\section{Tear Osmolarity}

Tear osmolarity has been widely studied both in research and clinical settings and is thought to represent one of the best global markers of DED. An insufficient or unstable tear film would by definition become hyperosmolar. The more widely available point-of-care test device uses micro-electrode technology to measure the number of charged particles in a tear sample provide an estimate of the tear osmolarity. Normal tear osmolarity has a value of 302 $\mathrm{mOsm} / \mathrm{L}$, with minimal inter-eye difference. A value of $308 \mathrm{mOsm} / \mathrm{L}$ in either eye is often used as the threshold in differentiating normal and early stages of DED, with $316 \mathrm{mOsm} / \mathrm{L}$ used a cutoff for more advanced DED. ${ }^{1}$ An important characteristic of tear osmolarity is its variability, both inter-eye as well as repeat measurements in the same eye. The worse the severity of dry eyes, the more variable tear osmolarity has been found to be $(6.9 \pm 5.9$ $\mathrm{mOsm} / \mathrm{L}$ in mild, $11.7 \pm 10.9 \mathrm{mOsm} / \mathrm{L}$ in moderate, and $26.5 \pm 22.7 \mathrm{mOsm} / \mathrm{L}$ in severe DES, respectively). ${ }^{2}$ Thus, a difference of $8 \mathrm{mOsm} / \mathrm{L}$ between two eyes is also considered to be significant and compatible with an unstable tear film.

As noted earlier, given the variability of the results, there are patients with symptoms of DED whose tear osmolarity may be measured as normal. In other words, a normal value does not always rule out DED; and hence, an elevated tear osmolarity should not be considered a prerequisite for the diagnosis. However, an elevated osmolarity strongly suggests presence of an inadequate tear film compatible with DED. Furthermore, it is worth noting that osmolarity is best not used as a static measure (e.g. not like height measurement). Rather, in some ways, it is analogous to clinical tests such as blood glucose, where there can be moment to moment variability depending on the time of the day, the patient's food intake, physical activity, etc. The same way that the average blood sugar (Hemoglobin A1C) provides a more reliable measure of the patient's glucose control, in a patient with an unstable tear film, the average tear film osmolarity over a specific period would likely be elevated and thus a single measurement may not best reflect the overall status of the tear film. Therefore, by standardizing the clinical measurement to minimize the setting and operator variability, and by focusing on the trends and averages, tear osmolarity can offer valuable insights into the status of the tear film and potentially guide the status of therapy in many subtypes of DED.

\section{Inflammatory biomarkers}

Inflammation is a key driving mechanism in many cases of DED. However, differentiating cases of DED with a major inflammatory component from those in whom inflammation 
plays a less fundamental role can be challenging. Biomarkers that can detect subclinical inflammation and ideally, even provide information about the severity of inflammation, can significantly improve our ability to individualize therapies. One key inflammatory biomarker that is now in clinical use is matrix metalloproteinase (MMP)-9. This endopeptidase is part of the extracellular matrix remodeling that takes place after injury and has been found to be a key component of the inflammatory cycle in DED.

Quantitative assessment of MMP-9 levels seem to correlate well with DED. One study showed a level of $7.2 \mathrm{U} / \mathrm{mg}$ in controls, compared to $473 \mathrm{U} / \mathrm{mg}$ in patients with MGD, and $651 \mathrm{U} / \mathrm{mg}$ in patients with Sjogren's syndrome. ${ }^{3}$ However, qualitative measurements of MMP-9 levels have shown variable sensitivities and specificities, likely reflecting the myriad of etiologies leading to elevated inflammation. ${ }^{3-6}$ While it's not yet clear whether a negative qualitative test of MMP-9 is a reflection of lack of inflammation, stage of DED, or a cutoff value that is not sensitive enough, a positive MMP-9 test can certainly help guide treatment plan and support the use of anti-inflammatory therapy. ${ }^{7}$ In particular, a positive test would prompt the early use of anti-inflammatory medications, as outlined later in this review.

\section{Meibomian gland Imaging}

Meibomian gland disease is a major, and perhaps the most common, etiologic factor in the pathogenesis of many subtypes of DED. Clinical diagnosis is often limited to examination of the lid margin by slit lamp to assess the degree of inspissation and telangiectasias, as well as subjective assessment of meibomian gland openings and meibum quality. However, information about the integrity of the glands within the tarsus has generally been more cumbersome to obtain using older meibography techniques. Recently, infrared based noncontact imaging modalities of meibomian gland have offered detailed imaging to guide the diagnosis and treatment of MGD-related DED.

Infrared meibography utilizes non-contact methods to image both upper and lower lids. Meibomian gland dropout as assessed by this method correlates well with signs and symptoms of dry eye disease. ${ }^{8,9}$ The commercially available imaging systems in the U.S. utilize automated meibomian gland grading which further reduces the subjectivity of meibomian gland evaluation. ${ }^{10}$ Spectral domain ocular coherence tomography as well as confocal microscopy have similarly been used to evaluate meibomian gland function although they are less automated and less convenient. ${ }^{11-13}$

These imaging modalities can provide valuable objective information about the integrity of the glands, which in turn helps identify patients in whom MGD is an underlying cause of their DED and thus guide appropriate therapy.

\section{Tear film stability and volume}

Traditionally, tear film stability and volume/production are assessed by fluorescein tear breakup time (TBUT) and Schirmer testing. While these tests remain essential components of the ocular surface exam, they are subjective and are influenced by many factors, including fluorescein volume. ${ }^{14}$ Several non-invasive tests now provide objective measure of these variables. 
Non-invasive Tear break-up time-Non-invasive measures of TBUT have been in practice for a long time and provide advantages over the fluorescein TBUT. Generally, these are topography-based imaging systems which provide automated measurement of TBUT using the distortion of the mires reflected from pre-corneal tear layer. ${ }^{9}$ Despite its advantages over fluorescein TBUT, particularly reduced variability and subjectivity, the use of non-invasive TBUT has not yet become a routine part of the DED exam and is limited mostly to clinical studies.

Lipid layer thickness-Another useful parameter in assessing tear film stability is the lipid layer thickness. Interferometry can offer a quantitative value of lipid layer thickness, providing insight into the health of the meibomian glands secretions While lipid layer thickness correlates well with symptoms as well as signs of dry eyes, ${ }^{9,15}$ it does not necessarily reflect quality of the lipid layer. ${ }^{16}$ More studies are needed to determine the precise role of this measure in the diagnosis and follow-up of patients with DED.

Tear Meniscus Height-Anterior segment optical coherence tomography (OCT), as well as some of the other ocular surface imaging systems that use interferometry, provide a noninvasive measure of the tear volume by quantifying the tear meniscus height. It has been shown to be a good proxy for tear volume and correlate with tear breakup time, corneal fluorescein staining, and diagnosis of DED. ${ }^{17}$ Despite its non-invasive nature, quantitative measurement of the tear meniscus height is generally not a part of the routine ocular exam in a DED. Anterior segment OCT, on the other hand, may be particularly useful for assessing and measuring conjunctivochalasis, a common finding in patients with ocular surface disease. ${ }^{18}$

\section{Advances in Dry Eye Therapeutics}

Treatment of DED is based on minimizing inflammation and optimizing the various components of the tear film. Artificial tears remain an essential part of patient comfort, with various lipid- and gel-based formulations holding promise in better simulating a healthy ocular surface. ${ }^{19}$ Other key interventions are listed below.

\section{Anti-inflammatory Therapies}

Inflammation is one of the major targets in treating DED and breaking the cycle of inflammation is crucial in improving symptoms. As noted above, the use of MMP-9 testing may help identify patients in whom anti-inflammatory therapy should be considered early. Regardless, all patients with DED deserve a trial of anti-inflammatory therapy at some point during their treatment.

Steroids-Corticosteroids are one of the most effective and rapid therapies available for suppressing inflammation on the ocular surface. In the context of DED, steroids are used mainly as pulse therapy. A short course of either commercially available topical steroids or preservative-free methylprednisolone $1 \%$ can be effective in improving DED and a positive response to steroids provides further evidence that inflammation likely plays a key role in the patient's disease. ${ }^{20,21}$ Long term therapy is obviously not a desirable option given the 
risk of complications, nonetheless, steroids are often helpful to "kick start" the antiinflammatory therapy with transition to the agents outlined below for long term therapy.

Cyclosporine A-One of the mainstays of anti-inflammatory therapy has been cyclosporine A (CsA). A meta-analysis of 12 randomized control trials comparing topical $0.05 \%$ to control showed improvement on Ocular Surface Disease Index (OSDI) scores, tear breakup time, Schirmer I scores, corneal fluorescein staining, and goblet cell densities. ${ }^{22}$ Despite compelling results in trials with subsequent FDA approval for keratoconjunctivitis sicca, in clinical practice, a large subgroup of patients do not respond to CsA $0.05 \%$. This may be taken as an indication that either inflammation does not play a critical role in many non-responders, or else $\mathrm{T}$ cells are not the main bad actors in those patients. Additional contributing factors to the lack of clinical response may be delayed onset of action and tolerability. Currently, different CsA preparations are in clinical trials for DED, and with improved tolerability and bio-availability, topical CsA is expected to remain an important non-steroidal option for controlling inflammation in DED.

Lifitegrast-The newest addition to the anti-inflammatory armamentarium for DED is the recently FDA approved drug, lifitegrast. ${ }^{23-25}$ It blocks lymphocyte-function associated antigen/intracellular adhesion molecule-1 (LFA-ICAM-1) interaction, thus decreasing T-cell recruitment. A notable advantage of lifitegrast appears to be its faster onset of action, with patients reporting improved symptoms within a few weeks. Clinical experience with lifitegrast is still quite limited, but with time, it should become another valuable tool for the management of inflammation in DED. An important question that will become apparent with further research is whether lifitegrast and CsA have any additive effects or if they are best used as single agents.

\section{Polyunsaturated fatty acids}

Omega-3 fatty acids have been shown to decrease inflammatory markers and ameliorate dry eye symptoms. Multiple trials have shown improved tear production, tear breakup time, Schirmer score, and OSDI scores. ${ }^{26-32}$ Likewise, studies have shown decreased HLA-DR positive cells, another marker of surface inflammation. ${ }^{26,31}$ While improvements have been shown in these trials, many of these supplements lack standardization to date. In particular, there may be significant differences in preparations that could affect the absorption and bioactivity of Omega-3. For instance, fish-oil based preparations provide different types of polyunsaturated fatty acids (EPA and DHA) compared to plant based preparations (ALA). Nevertheless, omega-3 supplement is a well-tolerated therapy to improve ocular surface health in nearly all forms of DED and is generally recommended to be used for all patients with no medical contraindications.

\section{Antibiotics with anti-inflammatory action}

Antibiotics, specifically those with concomitant anti-inflammatory action, play an important role in the management of DED due to MGD. The mechanism of action is generally twofold, first to reduce/alter the eyelid flora that is contributing to MGD and ocular surface inflammation, and second, through a direct anti-inflammatory effect. The two main groups of antibiotics that have been used are tetracyclines and macrolides. Oral doxycycline or 
minocycline successfully improve patient-reported symptoms and signs of MGD. ${ }^{33,34}$

However, patients are at risk for side effects, with gastrointestinal disturbance being the most common. Also, given the growing body of research on the role of gut microbiome in modulating the immune system, it is unclear what effects altering the gut flora with doxycycline has in the long term.

Azithromycin, either topical or oral, is used alternatively. A comparison study of topical azithromycin versus oral doxycycline showed that both improved signs and symptoms of MGD but with different compositions of changes in meibum, suggesting different mechanisms of action. ${ }^{35,36}$ Research suggests that unlike doxycycline, azithromycin can simulate meibomian gland cell differentiation in vitro. ${ }^{37}$ A 5-day course of oral azithromycin has also been studied in comparison to daily oral doxycycline and showed similar improvements but with less side effects at 2 months. ${ }^{36}$

\section{Meibomian gland heating/expression}

Intense Pulse Light therapy has been shown to be effective in dermatologic literature but studies in ophthalmic literature are still few. A prospective placebo-controlled study in patients with MGD showed improved subjective symptoms of DED in both the treatment and placebo eyes, but only the treatment eye showed improved lipid layer grade and tear break-up time over a 45 -day period. ${ }^{38}$ Other studies show similar improvement in subjective and objective measures. ${ }^{39,40}$ Combination therapy of intense pulsed light therapy and meibomian gland expression improved dry eye symptoms as well as meibomian gland function in a majority of patients. ${ }^{41}$ However, it is not without side effects: uveitis, iris atrophy, pupillary defects, photophobia, pain, and corneal pigment deposition have all been reported in patients who received IPL without appropriate eye protection. ${ }^{42-46}$

Thermal pulsation has also been used in patients with various degrees of MGD and shows improvement in patients, ${ }^{47-50}$ in some up to 3 years. ${ }^{51}$ However, the effects do fade away with time and an interventional study comparing single session of thermal pulsation to warm compresses twice daily for 3 months found that by 4 months, both groups show improved symptoms and signs without any significant difference between the two groups. ${ }^{52}$ Given the arduous nature of daily warm compresses, cost notwithstanding, thermal pulsation may be a good option for those with significant MGD related DED.

Overall, both therapies come with the advantage that they are a single-time intervention with longer lasting effects. While both are very promising, they are still relatively cost-prohibitive and not available as a treatment option for all patients.

\section{Therapies for Refractory cases of DED}

\section{Autologous Serum}

Autologous serum tears have long been used in a wide variety of ocular surface diseases, including DED. Autologous serum tears provide a natural substitute for the many bio-active proteins, vitamins and lipids that are typically present in human tears. Studies have shown conclusively that they can provide symptomatic relief in many subtypes of DED. ${ }^{53-59}$ Clinically, serum tears appear to be particularly useful in patients with marked aqueous tear 
deficiency. At our center, serum tears are routinely offered to patients who have failed standard measures. Our preferred starting concentration is $20 \%$, but studies comparing the different concentrations are limited. Platelet rich plasma tears, a closely related therapy, is thought to provide a richer concentration of growth factors, and likely has similar efficacy in this setting, but again studies comparing their efficacies in DED are lacking. ${ }^{60,61}$ Overall, autologous serum or plasma is an essential part of our therapeutic management of refractory cases. The major challenge with this therapeutic is accessibility and cost.

\section{Amniotic membrane}

Cryopreserved amniotic membrane transplantation has known anti-inflammatory and restorative properties in a variety of ocular surface disorders. It is believed to work through the presence of anti-inflammatory mediators within its stroma as well as its barrier properties by entrapping inflammatory cells. Both cryopreserved and freeze-dried amniotic membrane have been used as sutureless devices in patients with ocular surface disease. ${ }^{62}$ Given the limited number of studies its role in the management of DED is unclear.

\section{Contact Lenses}

With advancing technology, contact lenses have become a key therapeutic modality for DED, particularly for the severe cases. There are two types of lenses that are used. Soft bandage contact lenses have been studied in patients with a variety of ocular surface diseases with improvement in both subjective and objective measures. ${ }^{63-65}$ No infectious complications were noted when antibiotic prophylaxis was used along with extended wear. 63,64 Although studies are limited, in our clinical experience, in selected patients, soft contact lenses (daily or extended wear) can provide significant relief in refractory DED.

The most effective contact lens option for patients with severe DED is scleral lenses. Scleral lenses are typically fluid-filled and vault over the cornea, resting over the limbus, hence providing it with constant lubrication. Several studies using scleral lenses have shown improved comfort, decreased dry eye symptoms, and improved visual acuity with good safety profile in patients with severe ocular surface disease. ${ }^{66-71}$ While the PROSE (Prosthetic Replacement of Ocular Surface Ecosystem) lenses were the first lenses to be used for this indication, ${ }^{71}$ they are available only in select centers in the U.S. Newer scleral lenses are easier to fit and readily available commercially, making them more accessible to patients. Despite their efficacy, the use of scleral lenses remains limited partly due to availability and cost, and perhaps given the fact that many eye care providers may not be aware of their significant therapeutic benefits. One downside of scleral lenses is that they require more training to use and hence not all patients can handle them appropriately. With time, contact lenses are expected to play an increasing role in the management of refractory DED.

\section{Conclusion}

Overall, advances in technologies have significantly improved both diagnostics and therapeutics available for DED. Ongoing and future developments are expected to further enhance our ability to recognize, categorize and provide patient-specific therapies in DED. 


\section{Acknowledgements}

This work was supported by R01 EY024349-01A1 (ARD), Core grant EY01792 from NEI/NIH; unrestricted grant to the department from Research to Prevent Blindness (RPB). ARD is the recipient of a Career Development Award from RPB.

\section{References}

1. Bron AJ, Tomlinson A, Foulks GN, et al. Rethinking dry eye disease: a perspective on clinical implications. The ocular surface 2014;12(2 Suppl):S1-31. [PubMed: 24725379]

2. Lemp MA, Bron AJ, Baudouin C, et al. Tear osmolarity in the diagnosis and management of dry eye disease. American journal of ophthalmology 2011;151(5):792-798.e791. [PubMed: 21310379]

3. Solomon A, Dursun D, Liu Z, Xie Y, Macri A, Pflugfelder SC. Pro- and anti-inflammatory forms of interleukin-1 in the tear fluid and conjunctiva of patients with dry-eye disease. Investigative ophthalmology \& visual science 2001;42(10):2283-2292. [PubMed: 11527941]

4. Lanza NL, McClellan AL, Batawi H, et al. Dry Eye Profiles in Patients with a Positive Elevated Surface Matrix Metalloproteinase 9 Point-of-Care Test Versus Negative Patients. The ocular surface 2016;14(2):216-223. [PubMed: 26807724]

5. Messmer EM, von Lindenfels V, Garbe A, Kampik A. Matrix Metalloproteinase 9 Testing in Dry Eye Disease Using a Commercially Available Point-of-Care Immunoassay. Ophthalmology 2016;123(11):2300-2308. [PubMed: 27665213]

6. Schargus M, Ivanova S, Kakkassery V, Dick HB, Joachim S. Correlation of Tear Film Osmolarity and 2 Different MMP-9 Tests With Common Dry Eye Tests in a Cohort of Non-Dry Eye Patients. Cornea 2015;34(7):739-744. [PubMed: 25909238]

7. Sambursky R Presence or absence of ocular surface inflammation directs clinical and therapeutic management of dry eye. Clinical ophthalmology (Auckland, N.Z.) 2016;10:2337-2343.

8. Finis D, Ackermann P, Pischel N, et al. Evaluation of Meibomian Gland Dysfunction and Local Distribution of Meibomian Gland Atrophy by Non-contact Infrared Meibography. Current eye research 2015;40(10):982-989. [PubMed: 25330304]

9. Ji YW, Lee J, Lee H, Seo KY, Kim EK, Kim TI. Automated Measurement of Tear Film Dynamics and Lipid Layer Thickness for Assessment of Non-Sjogren Dry Eye Syndrome With Meibomian Gland Dysfunction. Cornea 2017;36(2):176-182. [PubMed: 28060064]

10. Ngo W, Srinivasan S, Schulze M, Jones L. Repeatability of grading meibomian gland dropout using two infrared systems. Optometry and vision science : official publication of the American Academy of Optometry 2014;91(6):658-667. [PubMed: 24830370]

11. Liang Q, Pan Z, Zhou M, et al. Evaluation of Optical Coherence Tomography Meibography in Patients With Obstructive Meibomian Gland Dysfunction. Cornea 2015;34(10):1193-1199. [PubMed: 26226467]

12. Yoo YS, Na KS, Byun YS, et al. Examination of Gland Dropout Detected on Infrared Meibography by Using Optical Coherence Tomography Meibography. The ocular surface 2017;15(1):130-138.e131. [PubMed: 27816570]

13. Kobayashi A, Yoshita T, Sugiyama K. In vivo findings of the bulbar/palpebral conjunctiva and presumed meibomian glands by laser scanning confocal microscopy. Cornea 2005;24(8):985-988. [PubMed: 16227847]

14. Greiner JV, Finnemore VM, Exford JM, et al. Effects of fluorescein instillation methods on the tear film lipid layer. Advances in experimental medicine and biology 2002;506(Pt A):507-512. [PubMed: 12613953]

15. Blackie CA, Solomon JD, Scaffidi RC, Greiner JV, Lemp MA, Korb DR. The relationship between dry eye symptoms and lipid layer thickness. Cornea 2009;28(7):789-794. [PubMed: 19574906]

16. King-Smith PE, Reuter KS, Braun RJ, Nichols JJ, Nichols KK. Tear film breakup and structure studied by simultaneous video recording of fluorescence and tear film lipid layer images. Investigative ophthalmology \& visual science 2013;54(7):4900-4909. [PubMed: 23766476] 
17. Ibrahim OM, Dogru M, Takano Y, et al. Application of visante optical coherence tomography tear meniscus height measurement in the diagnosis of dry eye disease. Ophthalmology 2010;117(10): 1923-1929. [PubMed: 20605216]

18. Gumus K, Pflugfelder SC. Increasing prevalence and severity of conjunctivochalasis with aging detected by anterior segment optical coherence tomography. American journal of ophthalmology 2013;155(2):238-242.e232. [PubMed: 23036571]

19. Baudouin C, Galarreta DJ, Mrukwa-Kominek E, et al. Clinical evaluation of an oil-based lubricant eyedrop in dry eye patients with lipid deficiency. European journal of ophthalmology 2017;27(2): 122-128. [PubMed: 27791253]

20. Marsh P, Pflugfelder SC. Topical nonpreserved methylprednisolone therapy for keratoconjunctivitis sicca in Sjogren syndrome. Ophthalmology 1999;106(4):811-816. [PubMed: 10201607]

21. Hong S, Kim T, Chung SH, Kim EK, Seo KY. Recurrence after topical nonpreserved methylprednisolone therapy for keratoconjunctivitis sicca in Sjogren's syndrome. Journal of ocular pharmacology and therapeutics : the official journal of the Association for Ocular Pharmacology and Therapeutics 2007;23(1):78-82.

22. Wan KH, Chen LJ, Young AL. Efficacy and Safety of Topical $0.05 \%$ Cyclosporine Eye Drops in the Treatment of Dry Eye Syndrome: A Systematic Review and Meta-analysis. The ocular surface 2015;13(3):213-225. [PubMed: 26045239]

23. Holland EJ, Luchs J, Karpecki PM, et al. Lifitegrast for the Treatment of Dry Eye Disease: Results of a Phase III, Randomized, Double-Masked, Placebo-Controlled Trial (OPUS-3). Ophthalmology 2017;124(1):53-60. [PubMed: 28079022]

24. Sheppard JD, Torkildsen GL, Lonsdale JD, et al. Lifitegrast ophthalmic solution $5.0 \%$ for treatment of dry eye disease: results of the OPUS-1 phase 3 study. Ophthalmology 2014;121(2):475-483. [PubMed: 24289915]

25. Tauber J, Karpecki P, Latkany R, et al. Lifitegrast Ophthalmic Solution 5.0\% versus Placebo for Treatment of Dry Eye Disease: Results of the Randomized Phase III OPUS-2 Study. Ophthalmology 2015;122(12):2423-2431. [PubMed: 26365210]

26. Brignole-Baudouin F, Baudouin C, Aragona P, et al. A multicentre, double-masked, randomized, controlled trial assessing the effect of oral supplementation of omega- 3 and omega- 6 fatty acids on a conjunctival inflammatory marker in dry eye patients. Acta ophthalmologica 2011;89(7):e591597. [PubMed: 21834921]

27. Deinema LA, Vingrys AJ, Wong CY, Jackson DC, Chinnery HR, Downie LE. A Randomized, Double-Masked, Placebo-Controlled Clinical Trial of Two Forms of Omega-3 Supplements for Treating Dry Eye Disease. Ophthalmology 2017;124(1):43-52. [PubMed: 27817918]

28. Epitropoulos AT, Donnenfeld ED, Shah ZA, et al. Effect of Oral Re-esterified Omega-3 Nutritional Supplementation on Dry Eyes. Cornea 2016;35(9):1185-1191. [PubMed: 27442314]

29. Georgakopoulos CD, Makri OE, Pagoulatos D, et al. Effect of Omega-3 Fatty Acids Dietary Supplementation on Ocular Surface and Tear Film in Diabetic Patients with Dry Eye. Journal of the American College of Nutrition 2017;36(1):38-43. [PubMed: 27797641]

30. Qiao J, Yan X. Emerging treatment options for meibomian gland dysfunction. Clinical ophthalmology (Auckland, N.Z.) 2013;7:1797-1803.

31. Sheppard JD Jr., Singh R, McClellan AJ, et al. Long-term Supplementation With n-6 and n-3 PUFAs Improves Moderate-to-Severe Keratoconjunctivitis Sicca: A Randomized Double-Blind Clinical Trial. Cornea 2013;32(10):1297-1304. [PubMed: 23884332]

32. Zhu W, Wu Y, Li G, Wang J, Li X. Efficacy of polyunsaturated fatty acids for dry eye syndrome: a meta-analysis of randomized controlled trials. Nutrition reviews 2014;72(10):662-671. [PubMed: 25236365]

33. Wladis EJ, Bradley EA, Bilyk JR, Yen MT, Mawn LA. Oral Antibiotics for Meibomian GlandRelated Ocular Surface Disease: A Report by the American Academy of Ophthalmology. Ophthalmology 2016;123(3):492-496. [PubMed: 26707417]

34. Yoo SE, Lee DC, Chang MH. The effect of low-dose doxycycline therapy in chronic meibomian gland dysfunction. Korean journal of ophthalmology : KJO 2005;19(4):258-263. [PubMed: 16491814] 
35. Foulks GN, Borchman D, Yappert M, Kakar S. Topical azithromycin and oral doxycycline therapy of meibomian gland dysfunction: a comparative clinical and spectroscopic pilot study. Cornea 2013;32(1):44-53. [PubMed: 22668581]

36. Kashkouli MB, Fazel AJ, Kiavash V, Nojomi M, Ghiasian L. Oral azithromycin versus doxycycline in meibomian gland dysfunction: a randomised double-masked open-label clinical trial. The British journal of ophthalmology 2015;99(2):199-204. [PubMed: 25138765]

37. Liu Y, Kam WR, Ding J, Sullivan DA. Can tetracycline antibiotics duplicate the ability of azithromycin to stimulate human meibomian gland epithelial cell differentiation? Cornea 2015;34(3):342-346. [PubMed: 25611398]

38. Craig JP, Chen YH, Turnbull PR. Prospective trial of intense pulsed light for the treatment of meibomian gland dysfunction. Investigative ophthalmology \& visual science 2015;56(3):19651970. [PubMed: 25678687]

39. Gupta PK, Vora GK, Matossian C, Kim M, Stinnett S. Outcomes of intense pulsed light therapy for treatment of evaporative dry eye disease. Canadian journal of ophthalmology. Journal canadien d'ophtalmologie 2016;51(4):249-253.

40. Toyos R, McGill W, Briscoe D. Intense pulsed light treatment for dry eye disease due to meibomian gland dysfunction; a 3-year retrospective study. Photomedicine and laser surgery 2015;33(1):41-46. [PubMed: 25594770]

41. Vegunta S, Patel D, Shen JF. Combination Therapy of Intense Pulsed Light Therapy and Meibomian Gland Expression (IPL/MGX) Can Improve Dry Eye Symptoms and Meibomian Gland Function in Patients With Refractory Dry Eye: A Retrospective Analysis. Cornea 2016;35(3):318-322. [PubMed: 26785301]

42. Crabb M, Chan WO, Taranath D, Huilgol SC. Intense pulsed light therapy (IPL) induced iritis following treatment for a medial canthal capillary malformation. The Australasian journal of dermatology 2014;55(4):289-291. [PubMed: 24417640]

43. Hong S, Lee JR, Lim T. Pigment deposition of cosmetic contact lenses on the cornea after intense pulsed-light treatment. Korean journal of ophthalmology : KJO 2010;24(6):367-370. [PubMed: 21165237]

44. Javey G, Schwartz SG, Albini TA. Ocular complication of intense pulsed light therapy: iris photoablation. Dermatologic surgery : official publication for American Society for Dermatologic Surgery [et al.] 2010;36(9):1466-1468.

45. Jewsbury H, Morgan F. Uveitis and iris photoablation secondary to intense pulsed light therapy. Canadian journal of ophthalmology. Journal canadien d'ophtalmologie 2012;47(4):e13-14.

46. Lee WW, Murdock J, Albini TA, O’Brien TP, Levine ML. Ocular damage secondary to intense pulse light therapy to the face. Ophthalmic plastic and reconstructive surgery 2011;27(4):263-265. [PubMed: 21346668]

47. Blackie CA, Coleman CA, Holland EJ. The sustained effect (12 months) of a single-dose vectored thermal pulsation procedure for meibomian gland dysfunction and evaporative dry eye. Clinical ophthalmology (Auckland, N.Z.) 2016;10:1385-1396.

48. Blackie CA, Carlson AN, Korb DR. Treatment for meibomian gland dysfunction and dry eye symptoms with a single-dose vectored thermal pulsation: a review. Current opinion in ophthalmology 2015;26(4):306-313. [PubMed: 26058030]

49. Finis D, Hayajneh J, Konig C, Borrelli M, Schrader S, Geerling G. Evaluation of an automated thermodynamic treatment (LipiFlow(R)) system for meibomian gland dysfunction: a prospective, randomized, observer-masked trial. The ocular surface 2014;12(2):146-154. [PubMed: 24725326]

50. Greiner JV. Long-term (12-month) improvement in meibomian gland function and reduced dry eye symptoms with a single thermal pulsation treatment. Clinical \& experimental ophthalmology 2013;41(6):524-530. [PubMed: 23145471]

51. Greiner JV. Long-Term (3 Year) Effects of a Single Thermal Pulsation System Treatment on Meibomian Gland Function and Dry Eye Symptoms. Eye \& contact lens 2016;42(2):99-107. [PubMed: 26222095]

52. Zhao Y, Veerappan A, Yeo S, et al. Clinical Trial of Thermal Pulsation (LipiFlow) in Meibomian Gland Dysfunction With Preteatment Meibography. Eye \& contact lens 2016;42(6):339-346. [PubMed: 26825281] 
53. Celebi AR, Ulusoy C, Mirza GE. The efficacy of autologous serum eye drops for severe dry eye syndrome: a randomized double-blind crossover study. Graefe's archive for clinical and experimental ophthalmology = Albrecht von Graefes Archiv fur klinische und experimentelle Ophthalmologie 2014;252(4):619-626.

54. Hussain M, Shtein RM, Sugar A, et al. Long-term use of autologous serum $50 \%$ eye drops for the treatment of dry eye disease. Cornea 2014;33(12):1245-1251. [PubMed: 25299423]

55. Hwang J, Chung SH, Jeon S, Kwok SK, Park SH, Kim MS. Comparison of clinical efficacies of autologous serum eye drops in patients with primary and secondary Sjogren syndrome. Cornea 2014;33(7):663-667. [PubMed: 24886995]

56. Liu Y, Hirayama M, Cui X, Connell S, Kawakita T, Tsubota K. Effectiveness of Autologous Serum Eye Drops Combined With Punctal Plugs for the Treatment of Sjogren Syndrome-Related Dry Eye. Cornea 2015;34(10):1214-1220. [PubMed: 26226470]

57. Mahelkova G, Vesela V, Seidler Stangova P, et al. [Tear Osmolarity in Patients with Severe Dry Eye Syndrome Before and After Autologous Serum Treatment: a Comparison with Tear Osmolarity in Healthy Volunteers]. Ceska a slovenska oftalmologie : casopis Ceske oftalmologicke spolecnosti a Slovenske oftalmologicke spolecnosti 2015;71(4):184-188. [PubMed: 26395851]

58. Pan Q, Angelina A, Marrone M, Stark WJ, Akpek EK. Autologous serum eye drops for dry eye. The Cochrane database of systematic reviews 2017;2:Cd009327. [PubMed: 28245347]

59. Urzua CA, Vasquez DH, Huidobro A, Hernandez H, Alfaro J. Randomized double-blind clinical trial of autologous serum versus artificial tears in dry eye syndrome. Current eye research 2012;37(8):684-688. [PubMed: 22670856]

60. Lopez-Plandolit S, Morales MC, Freire V, Grau AE, Duran JA. Efficacy of plasma rich in growth factors for the treatment of dry eye. Cornea 2011;30(12):1312-1317. [PubMed: 22012030]

61. Merayo-Lloves J, Sanchez-Avila RM, Riestra AC, et al. Safety and Efficacy of Autologous Plasma Rich in Growth Factors Eye Drops for the Treatment of Evaporative Dry Eye. Ophthalmic research 2016;56(2):68-73. [PubMed: 27229893]

62. Cheng AM, Zhao D, Chen R, et al. Accelerated Restoration of Ocular Surface Health in Dry Eye Disease by Self-Retained Cryopreserved Amniotic Membrane. The ocular surface 2016;14(1):5663. [PubMed: 26387870]

63. Inamoto Y, Sun YC, Flowers ME, et al. Bandage Soft Contact Lenses for Ocular Graft-versus-Host Disease. Biology of blood and marrow transplantation : journal of the American Society for Blood and Marrow Transplantation 2015;21(11):2002-2007.

64. Russo PA, Bouchard CS, Galasso JM. Extended-wear silicone hydrogel soft contact lenses in the management of moderate to severe dry eye signs and symptoms secondary to graft-versus-host disease. Eye \& contact lens 2007;33(3):144-147. [PubMed: 17502749]

65. Stoyanova EI, Otten HM, Wisse R, Rothova A, Riemens A. Bandage and scleral contact lenses for ocular graft-versus-host disease after allogeneic haematopoietic stem cell transplantation. Acta ophthalmologica 2015;93(7):e604. [PubMed: 25923173]

66. Alipour F, Kheirkhah A, Jabarvand Behrouz M. Use of mini scleral contact lenses in moderate to severe dry eye. Contact lens \& anterior eye : the journal of the British Contact Lens Association 2012;35(6):272-276. [PubMed: 22902924]

67. Carracedo G, Blanco MS, Martin-Gil A, Zicheng W, Alvarez JC, Pintor J. Short-term Effect of Scleral Lens on the Dry Eye Biomarkers in Keratoconus. Optometry and vision science : official publication of the American Academy of Optometry 2016;93(2):150-157. [PubMed: 26657697]

68. Tappin MJ, Pullum KW, Buckley RJ. Scleral contact lenses for overnight wear in the management of ocular surface disorders. Eye (London, England) 2001;15(Pt 2):168-172.

69. Weber SL, de Souza RB, Gomes JA, Hofling-Lima AL. The Use of the Esclera Scleral Contact Lens in the Treatment of Moderate to Severe Dry Eye Disease. American journal of ophthalmology 2016;163:167-173.e161. [PubMed: 26701271]

70. Yuksel E, Bilgihan K, Novruzlu S, Yuksel N, Koksal M. The Management of Refractory Dry Eye With Semi-Scleral Contact Lens. Eye \& contact lens 2016.

71. Dimit R, Gire A, Pflugfelder SC, Bergmanson JP. Patient ocular conditions and clinical outcomes using a PROSE scleral device. Contact lens \& anterior eye : the journal of the British Contact Lens Association 2013;36(4):159-163. [PubMed: 23499361] 


\section{Highlights}

This review covers the latest diagnostic and therapeutic strategies in the management of dry eye disease. New diagnostic tests including tear film osmolarity, inflammatory biomarkers and meibomian gland imaging have enhanced our ability to recognize and categorize patients with dry eye disease. Therapeutically, anti-inflammatory therapy, meibomian gland heating and expression, and scleral contact lenses are some of the latest options available for treating dry eye disease. 